\title{
Ventrículo izquierdo no compacto
}

\author{
Left ventricular noncompaction
}

\author{
Erik Trespalacios, Alfonso Mejía, Juan Manuel Senior • \\ Medellín (Colombia) - Brian Bergmark • Boston (EUA)
}

\section{Resumen}

El ventrículo izquierdo no compacto (VINC) o "esponjoso", es una cardiomiopatía recientemente descrita y reconocida con implicaciones pronósticas importantes. Se caracteriza por la presencia de un miocardio ancho con nichos intertrabeculares profundos sobre el lado endocavitario, llevando a hipertrabeculación. Aunque la etiología es desconocida, se ha descrito una importante agregación familiar y una superposición de esta patología con otras cardiopatías de carácter genético como la cardiomiopatía dilatada (CMD) y la cardiomiopatía hipertrófica $(\mathrm{CMH})$. Desde el punto de vista clínico se presenta como un síndrome de falla cardiaca, aunque puede debutar con muerte súbita, arritmias o embolismo sistémico. En la actualidad la herramienta diagnóstica de mayor utilidad es la ecocardiografía, por sus menores costos, reproducibilidad y disponibilidad en forma extensa en los servicios médicos. Sin embargo, se ha demostrado una mayor certeza diagnóstica con la resonancia magnética cardiaca (RMC). El tratamiento de estos pacientes no difiere del recomendado para pacientes con síndrome de falla cardiaca de otras etiologías. (Acta Med Colomb 2011; 36: 187-195)

Palabras clave: ventrículo izquierdo no compacto, resonancia magnética cardiaca, ecocardiografía, genética, falla cardiaca, cardiopatías, cardiomiopatías.

\begin{abstract}
Left ventricular noncompaction (LVNC), or "spongy" cardiomyopathy, is a recently described and recognized finding with important prognostic implications. It is characterized by the presence of ventricular trabeculations and deep intertrabecular recesses on the endocavitary side, leading to hypertrabeculation. Although the etiology is unknown, a significant family aggregation of this disease as well as overlap with other genetic cardiomyopathies such as dilated cardiomyopathy and hypertrophic cardiomyopathy have been described. From the clinical point of view, LVNC often presents as heart failure, but may present with sudden death, arrhythmias and systemic embolism. At the present time the most useful diagnostic tool is transthoracic echocardiography because of its lower cost, reproducibility and availability, though cardiac magnetic resonance (CMR) imaging has shown greater diagnostic accuracy. Treatment of these patients is no different than that recommended for patients with heart failure syndromes of other etiologies. (Acta Med Colomb 2011; 36: 187-195)
\end{abstract}

Keywords: left ventricular noncompaction, cardiac magnetic resonance imaging, echocardiography, genetics, heart failure, heart disease, cardiomyopathy.
Dr. Trespalacios A Erik: Clínica de Falla Cardiaca, Universidad de Antioquia; Dr. Alfonso Mejía V.: Unidad Cardiovascular y de Trasplantes, Hospital Universitario San Vicente de Paúl; Dr. Juan Manuel Senior: FACP, Clínica de Falla Cardiaca, Universidad de Antioquia. Unidad Cardiovascular y de Trasplantes Hospital Universitario San Vicente de Paul. Medellín Colombia. Dr. Brian Bergmark: Harvard Medical School, Boston (EUA)

Correspondencia. Dr. Juan Manuel Senior S. Medellín (Colombia).

E-mail: mmbt@une.net.co.

Recibido: 25/III/2011 Aceptado: 06/X/2011

\section{Caso 1}

Paciente masculino de 38 años edad, natural, residente y procedente de Medellín (Antioquia). Con cuadro clínico de seis meses de evolución, consistente en deterioro de su clase funcional (NYHA I hasta IV), edema de miembros inferiores progresivo, disnea paroxística nocturna, ortopnea y palpitaciones. Sin antecedentes personales o familiares de relevancia, sin factores de riesgo cardiovascular. PA 100/60, FC 92 lpm, FR $22 \mathrm{rpm}$, temperatura $36^{\circ} \mathrm{C}$, con $\mathrm{SaO}_{2} 96 \%$ respirando aire ambiente, ingurgitación yugular a $45^{\circ}$, edema grado III, crépitos basales en campos pulmonares, ruidos cardíacos rítmicos, sin soplos, sin S3. Electrocardiograma con ritmo sinusal, bloqueo de rama izquierda del haz de His. Ecocardiograma deterioro de función sistólica con FEVI 22\%, dilatación de cavidades izquierdas, no patología valvular, se observó hipertrabeculación en las paredes del ventrículo izquierdo en el eje corto hacia el ápex y se calculó una relación de capa trabeculada sobre compacta de $>2$, cuando medido en sístole y en el Doppler color se logró observar llenado de los recesos de la zona trabeculada del ventrículo (Figura 1). Radiografía de tórax con cardiomegalia a expensas del ventrículo izquierdo, sin derrame pleural, 


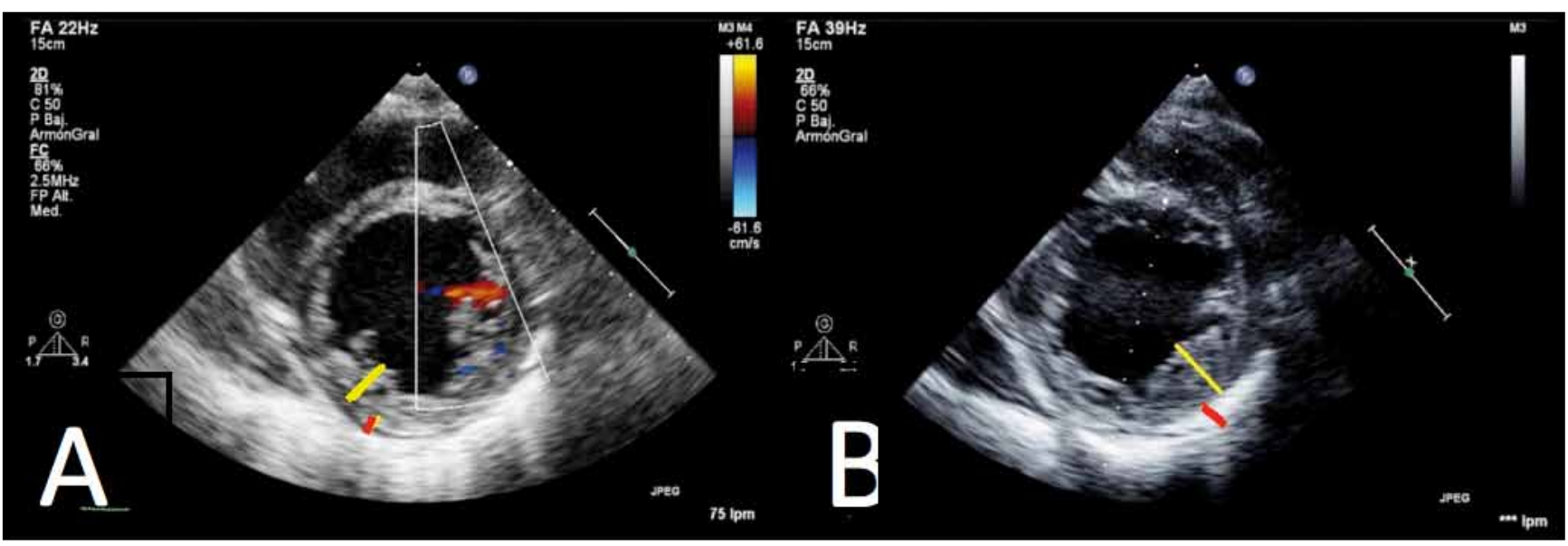

Figura 1. Imágenes tomadas por ecocardiografía transtorácica en modo $2 D$, en las que se observa una sección del eje corto en sus segmentos apicales. A) imagen con Doppler color que muestra sangre en la capa no compacta, con una comunicación de la cavidad ventricular y las trabeculas; B) imagen sin que muestra como la relación de la capa no compacta (línea amarilla) entre la compacta (línea roja) es $>2$.

congestión hiliar. En el laboratorio: hemograma normal, electrolitos normales, creatinina $0.7 \mathrm{mg} / \mathrm{dL}$, BUN $10 \mathrm{mg} /$ $\mathrm{dL}$, glucemia normal. Se realiza RMC que reporta hipertrabeculaciones comprometiendo principalmente la mitad apical del VI con una relación de capa trabeculada sobre compacta $>2.3$ medido en diástole y se hace diagnóstico de VINC (Figura 2). Como relataba palpitaciones se realizó Holter electrocardiográfico de 24 horas que demuestra episodios de taquicardia ventricular polimorfa no sostenida. Adicionalmente, se tomó biopsia endomiocárdica que fue reportada como tejido miocárdico normal. Se instauró manejo farmacológico con betabloqueadores, inhibidores de la enzima convertidora de angiotensina, ácido acetilsalicílico, diuréticos, con lo cual se produce mejoría clínica. Teniendo en cuenta el cuadro clínico, la fracción de eyección menor del $35 \%$, la dilatación ventricular, la presencia de disincronía eléctrica, la demostración de taquicardia ventricular, y la utilización de betabloqueadores, se solicitó implante de cardiorresincronizador; sin embargo, la aseguradora del paciente no aprobó el implante del dispositivo.

\section{Caso 2}

Paciente masculino de 30 años, quien consulta al servicio ambulatorio por disnea de esfuerzos (NYHA II), palpitaciones. Negaba otros síntomas. Sin factores de riesgo cardiovascular, sin historia familiar importante. PA 100/60, FC 68 lpm, FR $16 \mathrm{rpm}$, temperatura $36.5^{\circ} \mathrm{C}$, peso $78 \mathrm{~kg}$, auscultación cardiaca sin $\mathrm{S} 3$, sin soplos, punto de máximo impulso en línea axilar anterior, sin frote pericárdico. Auscultación pulmonar normal. Edema grado II en miembros inferiores. Resto del examen físico normal. La primera ecocardiografía de 2009 (en otra institución) con dilatación del VI (DFDVI 6.3 $\mathrm{cm}$ ), FEVI 35\%. Electrocardiograma con ritmo sinusal, PR corto pero sin onda delta. Radiografía de tórax cardiomegalia
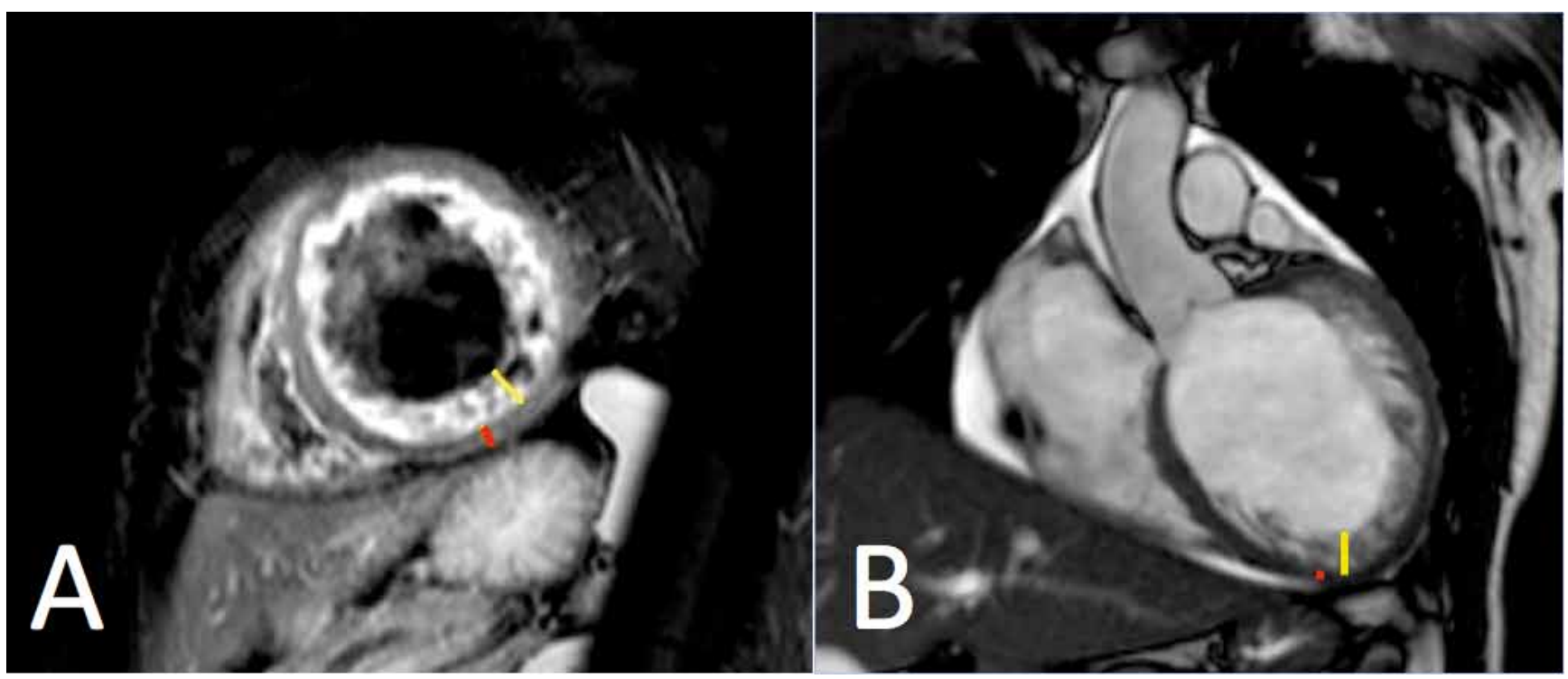

Figura 2. Imágenes tomadas con RMC. A) imagen del eje corto en la que se aprecia la relación entre la capa no compacta (línea amarilla) y la compacta (línea roja) > 2,3; B) imagen en la que se ve el VI, la válvula aortica y la raíz aórtica, claramente se observa una relación entre la capa no compacta y la compacta > 2,3. 
grado I a expensas del ventrículo izquierdo, no congestión hiliar, no derrame pleural. Laboratorio: creatinina $0.6 \mathrm{mg} /$ $\mathrm{dL}$, glucemia $88 \mathrm{mg} / \mathrm{dL}$, hemograma normal, electrolitos normales. HIV negativo, virus hepatitis negativos, ferritina en rango normal, serología para enfermedad de Chagas negativo, autoanticuerpos (anti-DNA, ANA, anti-Ro, anti-La, anti-Sm, anti-RNP) negativos, VDRL no reactivo.

Se repite ecocardiografía en 2010 (en nuestra institución) y muestra FEVI 20\%, Hipertrofia excéntrica del VI y signos de hipertrabeculación en el eje corto hacia el ápex, con una relación de capa trabeculada sobre compacta $>2$ medido en sístole, además en el Doppler color se observó que la zona trabeculada se llenaba con sangre proveniente del ventrículo, sin patología valvular (Figura 3). Se ordena RMC que reporta DFDVI $6.6 \mathrm{~cm}$, DFSVI $5.5 \mathrm{~cm}$, FEVI $30 \%$, hipertrabeculación del VI y se confirma una relación de la capa trabeculada sobre la compacta $>2.3$ medido en diástole (Figura 4). Se hace diagnóstico de VINC. Se inicia manejo con betabloqueadores, IECA, asa y se inicia rehabilitación cardiaca. Pasados seis meses el paciente alcanza clase funcional NYHA I con capacidad funcional de 8 METs en banda sin fin. Dado los hallazgos en el electrocardiograma se realiza estudio electrofisiológico en el cual no se documentaron alteraciones.

\section{Discusión}

El VINC aislado es un trastorno caracterizado por un miocardio ancho con nichos intratrabeculares profundos, y una pared miocárdica bilaminar con un estrato compacto y un estrato no compacto. Esta condición, descrita por primera vez por Engberding en 1984 (1) y en una serie de ocho casos publicada por Chin en 1990 (2), es clasificada por la American Heart Association como una cardiomiopatía genética primaria (3) y por la European Society of Cardiology (4) y
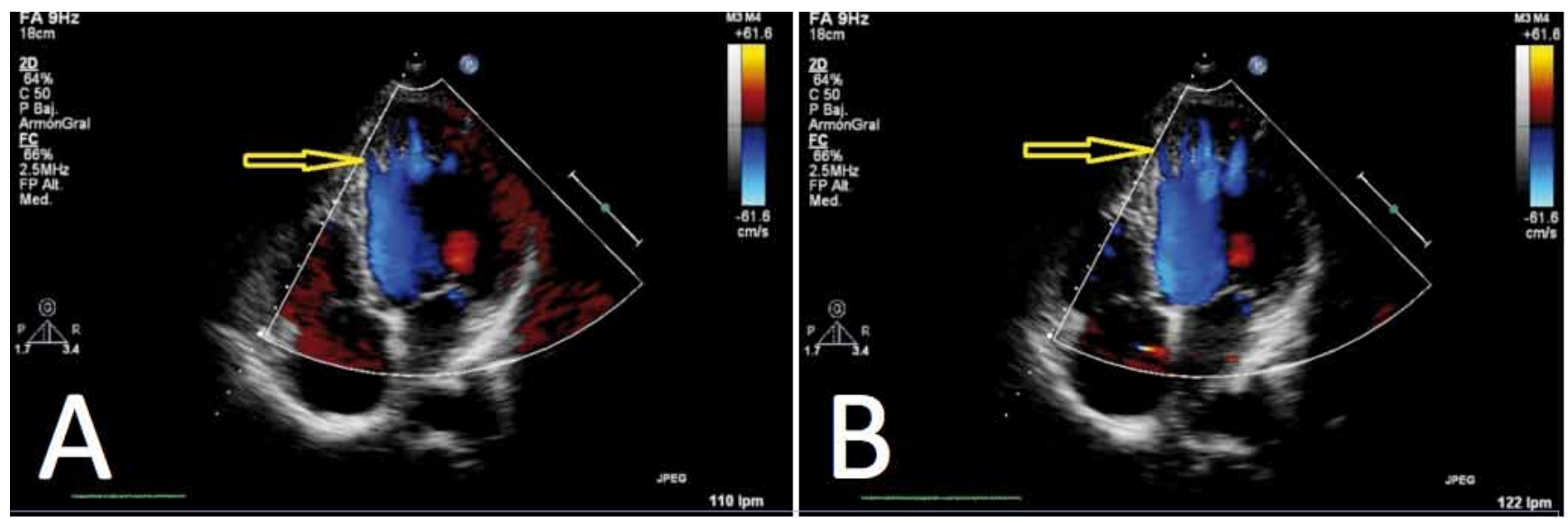

Figura 3. Imágenes tomadas por ecocardiografía transtorácica en modo $2 D$ con Doppler color, se observa una toma apical de cuatro cámaras. A) imagen en la que podemos observar como hay una continuidad entre la cavidad ventricular y la pared del VI (capa no compacta) en su zona apical (flecha); B) imagen en la que también pudimos verificar con Doppler color flujo de sangre en la pared ventricular

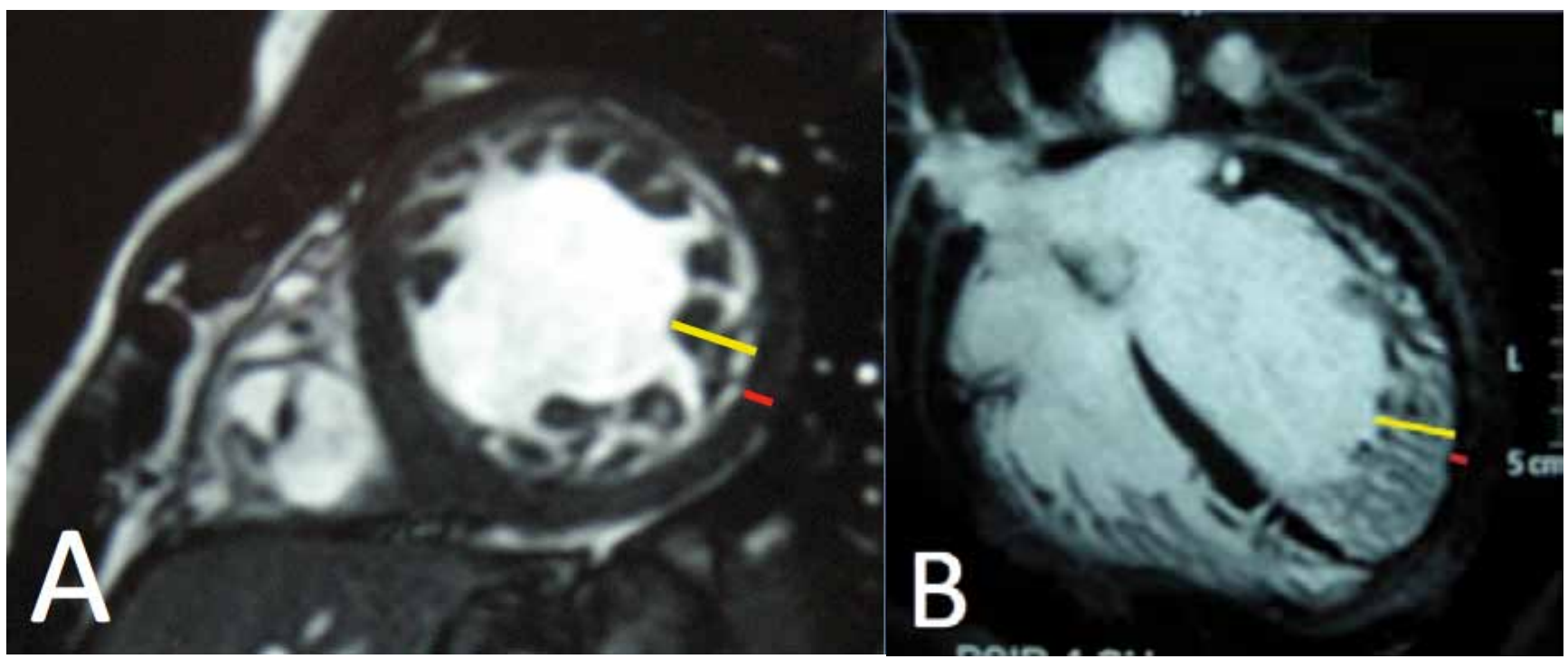

Figura 4. Imágenes tomadas con RMC. A) imagen del eje corto del VI en la que se observa una clara relación entre la capa no compacta (línea amarilla) y la capa compacta (línea roja) > $2.3 ; B$ ) imagen de cuatro cámaras en la que se aprecia la relación entre las capas no compacta y compacta del VI> 2.3. 
la World Health Organización (5) como una cardiomiopatía sin clasificación. Un intenso debate continúa con respecto a la definición del VINC como un trastorno primario, una respuesta secundaria, o una variante morfológica (6-8). Las características del VINC pueden coincidir con las de otras cardiomiopatías, incluso la CMH (especialmente la variante apical), la CMD y la cardiomiopatía restrictiva (9). Lo anterior contribuye a las dificultades en la clasificación (6), y hace que en algunas series hasta el $44 \%$ de los pacientes con VINC estén erróneamente diagnosticados como CMD o CMH $(10,11)$. Inicialmente, el VINC fue considerado bastante raro, con una prevalencia de $0.014 \%$ entre pacientes que acudían a un servicio de ecocardiografía (12), pero estudios más recientes informan prevalencias mayores (hasta $0.14 \%$ ) (11). Con el aumento de la conciencia de esta condición y la mayor capacidad de los métodos diagnósticos para definir el detalle de la anatomía cardiaca, ahora se estima que la prevalencia del VINC está alrededor del $3 \%$ entre pacientes con falla cardiaca que son remitidos a servicios de alta complejidad en países desarrollados (13). No se ha establecido con certeza la prevalencia del VINC entre la población general.

\section{Fisiopatología}

Originalmente el VINC era considerado el resultado de la falta de compactación del miocardio entre las semanas cinco y ocho de desarrollo embrionario (14). En la actualidad existe incertidumbre con respecto a esta teoría, y no está claro si una falla de compactación o alteraciones en la proliferación, diferenciación y maduración celular sean parte de la etiología dominante $(14,15)$. Una vez desarrollado, el VINC está asociado con alteraciones funcionales, incluso falla cardiaca sistólica y diastólica. Una teoría es que el VINC produce compromiso microcirculatorio con la consecuente isquemia y ésta causa, a su vez, fibrosis y remodelación miocárdica (16). En estudios con tomografía por emisión de positrones (PET) se ha demostrado menor reserva de flujo coronario, asociado con anormalidades del movimiento de la pared ventricular en pacientes con VINC, aunque éstos no cursen con enfermedad coronaria macrovascular $(14,16)$. Además, se ha observado menor metabolismo miocárdico aeróbico con mayor consumo de ácidos grasos en el miocardio de pacientes con VINC, quizás debido a isquemia localizada (17). Otras complicaciones del VINC incluyen arritmias y trombos intracavitarios (con los consecuentes fenómenos embólicos).

El VINC consiste en la persistencia del patrón fetal de la pared miocárdica. El miocardio fetal presenta una multitud de filamentos que cruzan de lado a lado de la cavidad cardiaca y éstos aproximadamente en la semana 5 a 8 inician un proceso de compactación (18), que culmina totalmente en el ventrículo izquierdo y parcialmente en el ventrículo derecho. Así que el hallazgo de estas trabeculaciones o "sinusoides" se considera patognomónico del VINC. El término "sinusoides intramiocárdicos persistentes" ha entrado en desuso porque se considera que éste describe una patología aparte que consiste en la persistencia de las trabeculaciones, igual que en el VINC, pero en la que también se demuestra comunicación de estos "sinusoides" con el sistema de arterias coronarias epicárdicas y que además tiene como factor etiológico la presencia de una obstrucción del tracto de salida del ventrículo izquierdo $(9,12)$.

\section{Genética}

A pesar de que una base genética para el VINC no ha sido demostrada para cada caso, aproximadamente el 18 a 33\% de éstos tienen asociación familiar (19). Existe una asociación entre algunos loci y el VINC, muchos de estos loci coinciden con los de otras cardiomiopatías, especialmente $\mathrm{CMH}$ (principalmente la variante apical), y también con otros loci importantes para enfermedades cardíacas congénitas $(20,21)$. Los productos de genes con asociaciones propuestas en el VINC incluyen la tafazzina (G4.5), alfadistrobrevina, proteínas de la sarcómera, proteínas del disco Z (como LIM domainbinding 3 y cypher/ZASP), canal de sodio tipo $V$ alfa (SCN5A), y FK binding protein-12 $(10,14,21)$.

El acortamiento de la tafazzina resulta en el síndrome Barth (cardiomiopatía, miopatía esquelética y neutropenia) y esto es debido a deficiencia de cardiolipina en la membrana mitocondrial (22). Entre pacientes con este trastorno ligado al sexo, el 53\% demostraron más trabeculación del ventrículo izquierdo o llenaron criterios para diagnóstico de VINC (23). La alfadistrobrevina es una proteína del citoesqueleto y está asociada con el VINC y otras enfermedades congénitas del corazón. Mutaciones en las proteínas sarcoméricas, incluso del gen de la actina cardiaca alfa (ACTC), se observan entre familias con VINC y CMH apical (3). Otras proteínas sarcoméricas asociadas con el VINC incluyen betamiosina de cadena pesada y troponina T2 (24).

Recomendaciones para la detección familiar y genética están en evolución. La Heart Failure Society of America recomienda la determinación de una historia familiar completa de tres generaciones y también la búsqueda de cardiomiopatía en parientes asintomáticos en primer grado de consanguinidad (25). El conocimiento acerca de etiologías genéticas específicas para el VINC es reciente aún y en la actualidad no existe una recomendación seria para la detección genética de familiares no afectados (25). Sin embargo, una publicación reciente apoya establecer el diagnóstico genético de pacientes con VINC (21).

\section{Epidemiología}

La prevalencia de esta patología no es enteramente conocida, principalmente en países en vía de desarrollo; aunque en países industrializados se ha podido documentar, en algunos servicios de cardiología de referencia, que ésta puede ir desde el $0.014 \%$ hasta el $0.14 \%(11,12,26)$. La edad promedio para el diagnóstico va de 37 a 42 años $(10,12)$, con rangos que se extienden desde la etapa neonatal hasta los 83 años. En las series publicadas hasta la fecha, los hombres 
en apariencia son más frecuentemente afectados que las mujeres, representando $56-82 \%$ de los casos $(2,12,26,27)$, incluso en nuestro reporte los dos casos son hombres. También se ha documentado una mayor prevalencia de hipertrabeculación del ventrículo izquierdo y VINC entre personas de raza negra, cuando se compara con caucásicos $(8,28)$, pero el significado patológico de este hallazgo no se ha dilucidado aún.

Un aspecto importante del comportamiento epidemiológico del VINC es su carácter genéticofamiliar y la posibilidad de tener agregación con otras cardiopatías determinadas genéticamente (9). Por ejemplo, en una serie de casos se documentó que hasta un $72 \%$ de los pacientes con VINC tenían historia familiar de CMH y muerte súbita; en esta misma serie el $25 \%$ de los familiares en primer grado de consanguinidad de los pacientes con VINC tenían alguna anormalidad en el ecocardiograma (10). En otra serie de casos con 57 pacientes con VINC, se logró evaluar ecocardiográficamente los familiares en primer grado de consanguinidad de 36 de estos pacientes, encontrándose 15 nuevos dianósticos de VINC (10 adultos y cinco niños) que se encontraban en 12 familias, en total un $33 \%$ de agregación familiar (11). Más recientemente, Hoedemaekers y cols. reportaron una serie de casos que incluyó 58 pacientes con diagnóstico de VINC (49 adultos y nueve niños) en la que encontraron que un 64\% de las familias de estos pacientes tenían una cardiomiopatía, ya sea VINC, CMH o CMD (21).

\section{Diagnóstico}

El diagnóstico del VINC está en evolución; las principales dificultades para llegar a un consenso en cuanto a los criterios diagnósticos son la carencia de un estándar claro de la lesión, la falta de estudios comparativos con la población general y el hecho de que muchos pacientes con diagnóstico de VINC tienen un curso clínico completamente asintomático $(8,11)$.

Las herramientas diagnósticas usadas en VINC son:

\section{Ecocardiografía}

En la actualidad es el método diagnóstico preferido $(14,29,30)$, y se han propuesto varios criterios diagnósticos $(15,31)$ (Tabla 1).

En un estudio reciente de casos y controles se evaluaron ecocardiográficamente 199 pacientes referidos a una clínica de falla cardiaca, encontrándose que 47 pacientes $(23.6 \%)$ tenían uno o más criterios de los descritos para el diagnóstico de VINC (8). De estos 47 pacientes, $78.7 \%$ llenaron los criterios diagnósticos propuestos por Chin y cols. (2), $63.8 \%$ los propuestos por Jenni y cols. (29) y $53.2 \%$ los propuestos por Stöllberger y cols. (32). Es de resaltar que si sólo una definición se hubiera usado, entonces la prevalencia en esta población habría variado de 12.1-18.6\% (8). Con lo anterior se demuestra que los criterios diagnósticos ecocardiográficos propuestos difieren mucho unos de otros y se necesita una revisión juiciosa de éstos. Además, en este mismo estudio, en la opinión del autor, puede implicar que la descripción
Tabla 1. Criterios ecocardiográficos para el diagnóstico de VINC.

\begin{tabular}{|c|c|}
\hline $\begin{array}{l}\text { Chin } \\
\text { et al. (2) }\end{array}$ & $\begin{array}{l}\text { VINC se define por relación } \mathrm{X} / \mathrm{Y} \leq 0.5 \text {. } \\
\mathrm{X}=\text { distancia desde la superficie epicárdica hasta el inicio de las } \\
\text { trabéculas. } \\
\mathrm{Y}=\text { distancia desde la superficie epicárdica hasta el pico de las tr } \\
\quad \text { béculas. } \\
\text { Estas medidas son tomadas en el ápex del VI de las vistas eje corto } \\
\text { paraesternal y apical y en la pared libre del VI al fin de la diástole. }\end{array}$ \\
\hline $\begin{array}{l}\text { Jenni } \\
\text { et al. (28) }\end{array}$ & $\begin{array}{l}\text { 1. Imagen del VI en la vista paraesternal del eje corto, tomada en } \\
\text { sístole. } \\
\text { VINC se define por relación } \mathrm{NC} / \mathrm{C}>2 \text {. } \\
\mathrm{NC}=\text { capa de miocardio no compacta. } \\
\mathrm{C}=\text { capa de miocardio compacta. } \\
\text { 2. Ausencia de anormalidades estructurales cardiacas. } \\
\text { 3. Trabeculaciones excesivamente prominentes y numerosas más } \\
\text { recesos intratrabeculares profundos. } \\
\text { 4. Recesos intratrabeculares que se llenan con sangre ventricular en } \\
\text { el Doppler color. }\end{array}$ \\
\hline $\begin{array}{l}\text { Stöllberger } \\
\text { et al. (30) }\end{array}$ & $\begin{array}{l}\text { 1. Más de tres trabeculaciones que protruyen desde la pared del VI, } \\
\text { apicalmente a los músculos papilares, visible en una imagen } \\
\text { planar simple. } \\
\text { 2. Espacios intertrabeculares perfundidos por sangre ventricular } \\
\text { visualizado con Doppler color. }\end{array}$ \\
\hline
\end{tabular}

de hipertrabeculaciones en un paciente con falla cardiaca no sea más que un hallazgo incidental (8).

\section{Resonancia magnética cardiaca (RMC)}

Es otro método para evaluar el VINC. En un estudio que usó la RMC establecieron una relación entre los estratos "no compacto" y "compacto" $\geq 2.3$ como el punto de corte, con lo anterior se consiguió una sensibilidad de $86 \%$, especificidad de $99 \%$, valor predictivo positivo de $75 \%$ y valor predictivo negativo de $99 \%$ para el diagnóstico de VINC (33); pero a diferencia de la ecocardiografía en la que las medidas se toman en sístole (29), en la RMC las medidas se toman al final de la diástole (33). Otro estudio evaluó la agudeza de la RMC para el diagnóstico de VINC; y encontró que si se usaba una relación entre los estratos "no compacto" y "compacto" > 2 se conseguía una sensibilidad del 100\% pero sólo un $67.5 \%$ de especificidad para detectar un VINC; si la relación se llevaba a $>2.3$ la sensibilidad era de $96.4 \%$ y la especificidad era de $81.2 \%$; pero cuando el punto de corte se llevó a 2.5 entonces la sensibilidad era de $97.4 \%$ y la especificidad de $96.4 \%$ (34).

Actualmente es considerado por muchos que la ecocardiografía es el método estándar para el diagnóstico de VINC, esto es producto de estudios en los que se observó una mayor agudeza diagnóstica con la ecocardiografía que con la RMC (35). Lo anterior es explicado porque la RMC era una técnica nueva y los protocolos para evaluar corazón eran pobres. Estudios más recientes han encontrado que la RMC tiene un poder superior a la ecocardiografía para detectar casos de VINC y para definir la extensión del compromiso anatómico (34,36-39). Recientemente ha sido propuesto un nuevo criterio diagnóstico para VINC, que permitiría diferenciar casos con hipertrabeculación normal de los reales casos con VINC; el criterio propuesto consiste 
en un cálculo con RMC del porcentaje de la masa ventricular que corresponde al estrato "no compacto", si ésta es > $20 \%$ se considera que corresponde a un caso verdadero de VINC, con lo que se consigue una sensibilidad de $78 \%$ y especificidad de $72 \%$ (38).

Aun habiéndose establecido con claridad que la RMC es una técnica más sensible y específica para el diagnóstico de VINC, su alto costo y limitado acceso hacen que deba ser utilizada como herramienta diagnóstica de segunda línea en pacientes con mala ventana acústica o con dudas diagnósticas, dejando a la ecocardiografía en la primera línea diagnóstica $(30,38,40)$.

Otras modalidades diagnósticas incluyen tomografía, ventriculografía contrastada (41), angioscopia con video, pero sin mayor utilidad por su pobre resolución anatómica (19). La radiografía de tórax puede aparecer normal en sujetos asintomáticos (2), también puede observarse cardiomegalia en el $86 \%$ de los pacientes y congestión puede ser evidente en pacientes con deterioro de la función sistólica del ventrículo izquierdo $(2,26)$.

\section{Clínica}

Los pacientes con diagnóstico de VINC pueden presentarse completamente asintomáticos o con condiciones que amenazan su vida (8). Las series más antiguas mostraban una mayor proporción de pacientes sintomáticos, pero las más recientes tienen pacientes con cursos clínicos más benignos (Tabla 2). Aunque los pacientes pueden presentarse con un espectro grande de síntomas, a continuación describiremos los más importantes por su frecuencia e impacto en la mortalidad y calidad de vida de los pacientes:

Falla cardiaca: la mayoría de pacientes se presentan con deterioro de la función sistólica, definida por ecocardiografía. Se ha encontrado que hasta $63 \%$ de los pacientes pueden tener una fracción de eyección del ventrículo izquierdo (FEVI) de $10 \%$ a $33 \%$, y hasta $86 \%$ de los pacientes pueden presentarse con una FEVI $<50 \%(2,8,11,12,26,9)$. Los pacientes pueden presentarse con clínica de falla cardiaca, habiéndose descrito que de $25 \%$ a $65 \%$ pueden tener una clase funcional NYHA I-II y del $35 \%$ al $53 \%$ pueden presentarse con clase funcional NYHA III-IV $(10-12,26,32,42)$. En un reporte de casos se encontró que los mejores predictores para disfunción sistólica del ventrículo izquierdo, en pacientes con VINC, fueron: número de segmentos afectados, edad de inicio de síntomas y la relación NC/C (11).

Arritmias: las arritmias ventriculares son las más frecuentemente reportadas, pudiéndose encontrar entre $6 \%$ y $63 \%$ de los pacientes $(2,10,12,26,43)$, y la característica más importante es que están asociadas con un gran riesgo de muerte súbita. En un reporte se documentó que los pacientes con TV tenían ventrículos más dilatados y FEVI más bajas, y no se encontró relación entre la extensión de las trabeculaciones y la presencia de TV (11). Aunque no hay estudios clínicos aleatorizados que hayan evaluado la utilidad del CDI en estos pacientes, sí existen series de casos que han reportado su uso con buenos resultados, tanto en

Tabla 2. En esta tabla: EKG anormal (*): porcentaje de pacientes de la población total que presentaron alguna anormalidad en el electrocardiograma; FC (口 falla cardiaca: porcentaje de pacientes de la población total que presentaron clínica compatible con falla cardiaca; FEVI $\downarrow$ ( $\square$ fracción de eyección del ventrículo izquierdo deteriorada: porcentaje de pacientes de la población total en los que se documentó un deterioro de la fracción de eyección del ventrículo izquierdo $(<35-40 \%) ;$ FEVI \% (\$) fracción de eyección del ventrículo izquierdo en porcentaje: es el promedio de la fracción de eyección del ventrículo izquierdo de la población total; MS (II) muerte súbita: porcentaje de los pacientes que murieron cuya causa fue muerte súbita; trombos (f) complicaciones tromboembólicas: porcentaje de pacientes de la población total que presentaron alguna complicación tromboembólica.

\begin{tabular}{|c|c|c|c|c|c|c|}
\hline \multicolumn{2}{|l|}{ Autor } & Chin et al(2). & Ichida et al. (26) & Oechslin et al. (11) & Murphy et al. (9) & Aras et al. (10) \\
\hline \multicolumn{2}{|c|}{ Fecha de publicación } & 1990 & 1999 & 2000 & 2005 & 2006 \\
\hline \multicolumn{2}{|l|}{ Población } & 8 & 27 & 34 & 45 & 67 \\
\hline \multicolumn{2}{|c|}{ Seguimiento en años } & 5 & $6(0,2-17)$ & $3,6 \pm 3,3$ & 46 & $30(9-50)$ \\
\hline \multicolumn{2}{|c|}{ Edad en años. } & $8.9(11-22)$ & $5(1-17)$ & $42 \pm 17$ & $37 \pm 17$ & $41 \pm 18$ \\
\hline \multicolumn{2}{|c|}{ Hombres \% } & 63 & 65,6 & 74 & $62 \%$ & $65,6 \%$ \\
\hline \multicolumn{2}{|c|}{ EKG anormal $(*)$} & $87,5 \%$ & $88 \%$ & $94 \%$ & $91 \%$ & $85 \%$ \\
\hline Clínica & 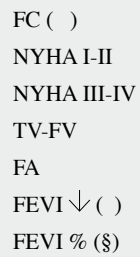 & $\begin{array}{c}63 \% \\
- \\
- \\
25 \% \\
25 \% \\
63 \% \\
-\end{array}$ & $\begin{array}{c}22 \% \\
- \\
- \\
- \\
3,7 \% \\
48 \% \\
61,5\end{array}$ & $\begin{array}{c}53 \% \\
65 \% \\
35 \% \\
41 \% \\
26 \% \\
86 \% \\
33 \pm 13\end{array}$ & $\begin{array}{c}65 \% \\
64 \% \\
36 \% \\
22 \% \\
6,6 \% \\
67 \% \\
-\end{array}$ & $\begin{array}{c}- \\
70 \% \\
30 \% \\
36 \% \\
14 \% \\
66 \% \\
35(20-48)\end{array}$ \\
\hline Desenlace & $\begin{array}{l}\text { Mortalidad } \\
\text { Trasplante } \\
\text { MS (II) } \\
\text { Trombos (I) }\end{array}$ & $\begin{array}{c}75 \% \\
- \\
35,3 \% \\
38 \%\end{array}$ & $\begin{array}{c}7,4 \% \\
3,7 \% \\
- \\
-\end{array}$ & \begin{tabular}{l|}
$35 \%$ \\
$12 \%$ \\
$50 \%$ \\
$24 \%$
\end{tabular} & $\begin{array}{c}2,2 \% \\
0 \\
100 \% \\
4 \%\end{array}$ & $\begin{array}{c}15 \% \\
0 \\
60 \% \\
9 \%\end{array}$ \\
\hline \multicolumn{2}{|c|}{ Agregación familiar } & $25 \%$ & $44 \%$ & $18 \%$ & $51 \%$ & $33 \%$ \\
\hline
\end{tabular}


prevención primaria como secundaria, sin diferencias importantes con respecto a los pacientes con otras etiologías de TV (10,12,44-46). Según Device Based Therapy guidelines el implante de un CDI, para la prevención de muerte súbita en estos pacientes, tiene una recomendación clase II con un nivel de evidencia B (45).

La FA también es frecuente en estos pacientes, presentándose entre 7-26\% de los casos (10-12), y la mayoría de los pacientes recibe un manejo convencional para la FA, igual que un paciente sin VINC. El bloqueo AV completo ha sido reportado en pacientes con VINC $(11,42,47-49)$, y el implante de un marcapasos se considera el tratamiento de elección (45), igual que en la población general. También, recientemente se ha reportado la asociación del VINC con el síndrome de Brugada (50); y en dos pacientes pediátricos, con el síndrome de QT largo (51).

El electrocardiograma es anormal en $85-94 \%$ de los casos (10-12,26,32). Puede encontrarse desviación del eje a la izquierda o a la derecha, onda $\mathrm{P}$ ancha o picuda, bloqueos AV de primer grado (o mayores) y defectos de conducción intraventriculares $(2,9,12,26)$.

Embolismo: este fenómeno tiene múltiples posibles etiologías, actualmente no se ha definido cuál es la responsable. Se han propuesto como probables causantes de los fenómenos embólicos las arritmias, la dilatación de cavidades izquierdas, y el movimiento lento de la sangre en los recesos intertrabeculares (30). Esta complicación se presenta entre 24 y $38 \%$ de los pacientes, pudiendo manifestarse como embolismos sistémicos o pulmonares $(2,9,11,12,26,30)$. En una serie que siguió a los pacientes por 10 años, se documentaron eventos embólicos en un 4\% de los pacientes y ninguno de ellos tenía documentada una FA (10), en otros reportes de casos se ha reportado un hallazgo similar (30).

Desenlaces: los pacientes pueden morir por múltiples causas; las más frecuentemente reportadas son arritmias ventriculares (TV-FV), falla cardiaca descompensada, y embolismos sistémicos (2). En una serie de casos se describió que, pasados cinco años del diagnóstico de VINC, $53 \%$ de los pacientes seguía vivo, $12 \%$ había recibido un trasplante cardiaco (por falla cardiaca) y 35\% había muerto; de los pacientes que murieron, la causa fue: $33 \%$ falla cardiaca, $50 \%$ muerte súbita, y $17 \%$ otras (ej. embolismo cerebral, embolismo intestinal, embolismo pulmonar, arritmias sin muerte súbita) (12). En el mismo registro, la probabilidad de sobrevida libre de eventos a los cinco años (combinado de muerte y trasplante cardiaco) fue de $58 \%$, con una edad media para muerte o trasplante cardiaco de 45 años. También se observó que no hubo deterioro de la función sistólica en los controles ecocardiográficos de 20 pacientes (en los que se realizó seguimiento ecocardiográfico) (12), hallazgos similares han sido reportados por otra serie (11). En otro registro, la sobrevida a seis años libre de eventos, fue de $47 \%$ con un claro peor pronóstico para pacientes sintomáticos (26). Lo anterior contrasta con series de casos más recientes, en las que el pronóstico de los pacientes con diagnóstico de VINC es mucho mejor, posiblemente reflejando la inclusión de pacientes en un periodo subclínico de la patología en estas nuevas series $(8,11)$.

En una serie de casos con 67 pacientes, con un seguimiento promedio a 30 meses (9-50 meses), se documentó una mortalidad de $15 \%$, y los factores que se relacionaron con mayor probabilidad de morir fueron: un mayor DFDVI (6.7 vs $5.6 \mathrm{~cm}, \mathrm{p}=0.006$ ), la FEVI más baja ( 28 vs $46 \%$, p $=0.001$ ), peor clase funcional (NYHA III-IV 86 vs $21 \%, \mathrm{p}$ $=0.002)$, y la presencia de TV (60 vs $24 \%, \mathrm{p}=0.03)$. Sin embargo, ninguno de los siguientes fueron buenos predictores de mortalidad: edad, sexo, relación $\mathrm{NC} / \mathrm{C}$, número de segmentos afectados, ritmo distinto al sinusal o bloqueos de rama (11).

\section{Manejo}

No existen estudios que hayan evaluado la respuesta de estos pacientes a diferentes modalidades de tratamiento. Según los reportes de casos y las series publicadas, el manejo que se da es el mismo que se recomienda para los demás pacientes con falla cardiaca; entonces IECA-ARA II, betabloqueadores, diuréticos, digitálicos, antagonistas de la aldosterona, terapia de resincronización cardiaca y trasplante cardiaco son las opciones terapéuticas disponibles para los pacientes con VINC $(10,11,42,52-54)$.

Ha sido reportado el uso de anticoagulación para prevención primaria y secundaria de eventos embólicos (2). Según una serie importante de pacientes la anticoagulación podría reducir la incidencia de eventos cardioembólicos, principalmente en pacientes con función sistólica severamente deteriorada (FEVI < 25\%) (10). Algunos prefieren reservar la anticoagulación para pacientes con historia de fibrilación auricular, ventrículos severamente dilatados e historia de eventos cardioembólicos y dar a los demás pacientes prevención primaria con aspirina (11).

Las arritmias han sido manejadas tal como se recomienda para pacientes con falla cardiaca en general. Importante mención merece la alta frecuencia de TV en este grupo de pacientes, requiriendo en muchos casos el uso de CDI, dado que están asociadas con alta probabilidad de muerte súbita $(11,42,53)$. Algunos recomiendan también la realización de una evaluación con Holter electrocardiográfico cada año para detectar arritmias (53).

Los pacientes que reciben un diagnóstico de VINC requieren evaluación genética y consejería genética. Además, los familiares en primer grado de consanguinidad deben ser evaluados, dado el carácter hereditario y la alta agregación familiar demostrada $(11,21)$.

\section{Conclusiones}

El VINC es una patología de relativa reciente descripción. Con la mayor conciencia de los médicos acerca de su existencia, ha aumentado el número de pacientes reportados y la prevalencia descrita ha aumentado. Existen aún dificultades para una correcta clasificación de un caso con hipetrabecu- 
laciones como VINC o como una variante normal. Además, existe cierta superposición con otras patologías determinadas genéticamente, como la CMD y la $\mathrm{CMH}$, tanto que casi la mitad de los pacientes que son diagnosticados con un VINC estaban clasificados erróneamente como CMH o CMD. Otro punto importante es que si bien disponemos de varias propuestas para el diagnóstico ecocardiográfico y por RMC, aún no sabemos cuál de éstas es la más apropiada. Aun con todas estas dificultades, es relevante establecer el diagnóstico de VINC porque tiene implicaciones pronósticas importantes, y también requiere manejo dirigido a complicaciones puntuales. El tratamiento no difiere del recomendado para pacientes con síndrome de falla cardiaca, incluyendo la terapia de resincronización y el trasplante cardiaco.

\section{Glosario}

Cardiodesfibrilador implantable: CDI; cardiomiopatía hipertrófica: $\mathrm{CMH}$; cardiomiopatía dilatada: $\mathrm{CMD}$; diámetro de fin de diástole del ventrículo izquierdo: DFDVI; diámetro de fin de sístole del ventrículo izquierdo: DFSVI; fibrilación auricular: FA; fracción de eyección del ventrículo izquierdo: FEVI; resonancia magnética cardiaca: RMC; taquicardia ventricular: TV; terapia de resincronización cardiaca: TRC; ventrículo izquierdo: VI

\section{Conflictos de interés}

Ninguno.

\section{Referencias}

1. Engberding R, Bender F. Identification of a rare congenital anomaly of the myocardium by two-dimensional echocardiography: persistence of isolated myocardial sinusoids. Am J Cardiol 1984; 53:1733-4.

2. Chin TK, Perloff JK, Williams RG, Jue K, Mohrmann R. Isolated noncompaction of left ventricular myocardium. A study of eight cases. Circulation 1990; 82: 507-13.

3. Maron BJ, Towbin JA, Thiene G, Antzelevitch C, Corrado D, Arnett D, et al. Contemporary definitions and classification of the cardiomyopathies: an American Heart Association Scientific Statement from the Council on Clinical Cardiology, Heart Failure and Transplantation Committee; Quality of Care and Outcomes Research and Functional Genomics and Translational Biology Interdisciplinary Working Groups; and Council on Epidemiology and Prevention. Circulation 2006. 1807-16.

4. Elliott P, Andersson B, Arbustini E, Bilinska Z, Cecchi F, Charron P, et al. Classification of the cardiomyopathies: a position statement from the European Society Of Cardiology Working Group on Myocardial and Pericardial Diseases. Eur Heart J 2008. p. 270-6.

5. Richardson P, McKenna W, Bristow M, Maisch B, Mautner B, O'Connell J, et al. Report of the 1995 World Health Organization/International Society and Federation of Cardiology Task Force on the Definition and Classification of cardiomyopathies. Circulation 1996; 93: 841-2.

6. Biagini E, Ragni L, Ferlito M, Pasquale F, Lofiego C, Leone O, et al. Different types of cardiomyopathy associated with isolated ventricular noncompaction. Am J Cardiol 2006. p. 821-4.

7. Sen-Chowdhry S, McKenna WJ. Left ventricular noncompaction and cardiomyopathy: cause, contributor, or epiphenomenon? Curr Opin Cardiol 2008. p. 171-5.

8. Kohli SK, Pantazis AA, Shah JS, Adeyemi B, Jackson G, McKenna WJ, et al. Diagnosis of left-ventricular non-compaction in patients with left-ventricular systolic dysfunction: time for a reappraisal of diagnostic criteria? Eur Heart J England: 2008. p. 89-95.

9. Martínez-Baca López F, Alonso Bravo RM, Rodríguez Huerta DA. Características ecocardiográficas da cardiomiopatia não-compactada: diagnóstico perdido ou errôneo. Arq Bras Card 2009; 93: e33-e35.

10. Murphy RT, Thaman R, Blanes JG, Ward D, Sevdalis E, Papra E, et al. Natural history and familial characteristics of isolated left ventricular non-compaction. Eur Heart J England: 2005. p. 187-92.

11. Aras D, Tufekcioglu O, Ergun K, Ozeke O, Yildiz A, Topaloglu S, et al. Clinical features of isolated ventricular noncompaction in adults long-term clinical course, echocardiographic properties, and predictors of left ventricular failure.J Card Fail United States: 2006. p. 726-33.

12. Oechslin EN, Attenhofer Jost CH, Rojas JR, Kaufmann PA, Jenni R. Longterm follow-up of 34 adults with isolated left ventricular noncompaction: a distinct cardiomyopathy with poor prognosis. J Am Coll Cardiol United States: 2000. p. 493-500.

13. Kovacevic-Preradovic T, Jenni R, Oechslin EN, Noll G, Seifert B, Attenhofer Jost CH. Isolated left ventricular noncompaction as a cause for heart failure and heart transplantation: a single center experience. Cardiology Switzerland: 2008 S. Karger AG, Basel.; 2009. p. 158-64

14. Engberding R, Yelbuz TM, Breithardt G. Isolated noncompaction of the left ventricular myocardium -- a review of the literature two decades after the initial case description. Clin Res Cardiol 2007; 96: 481-8.

15. Stollberger C, Finsterer J. Trabeculation and left ventricular hypertrabeculation noncompaction. J Am Soc Echocardiogr. United States: 2004. p. 1120-1.

16. Jenni R, Wyss CA, Oechslin EN, Kaufmann PA. Isolated ventricular noncompaction is associated with coronary microcirculatory dysfunction. J Am Coll Cardiol. United States: 2002. p. 450-4

17. Toyono M, Kondo C, Nakajima Y, Nakazawa M, Momma K, Kusakabe K. Effects of carvedilol on left ventricular function, mass, and scintigraphic findings in isolated left ventricular non-compaction. Heart 2001; 86:E4

18. Weiford BC, Subbarao VD, Mulhern KM. Noncompaction of the ventricular myocardium. Circulation 2004. p. 2965-71

19. Sarma RJ, Chana A, Elkayam U. Left ventricular noncompaction. Prog Cardiovasc Dis United States: 2010. p. 264-73.

20. Kelley-Hedgepeth A, Towbin JA, Maron MS. Images in cardiovascular medicine. Overlapping phenotypes: left ventricular noncompaction and hypertrophic cardiomyopathy. Circulation United States: 2009. p. e588-9.

21. Hoedemaekers YM, Caliskan K, Michels M, Frohn-Mulder I, van der Smagt JJ, Phefferkorn JE, et al. The importance of genetic counseling, DNA diagnostics, and cardiologic family screening in left ventricular noncompaction cardiomyopathy. Circ Cardiovasc Genet United States: 2010. p. 232-9.

22. Bleyl SB, Mumford BR, Brown-Harrison MC, Pagotto LT, Carey JC, Pysher TJ, et al. Xq28-linked noncompaction of the left ventricular myocardium: prenatal diagnosis and pathologic analysis of affected individuals. Am J Med Genet United States: 1997. p. 257-65.

23. Spencer CT, Bryant RM, Day J, Gonzalez IL, Colan SD, Thompson WR, et al. Cardiac and clinical phenotype in Barth syndrome. Pediatrics United States: 2006. p. e337-46.

24. Luedde M, Ehlermann P, Weichenhan D, Will R, Zeller R, Rupp S, et al Severe familial left ventricular non-compaction cardiomyopathy due to a novel troponin T (TNNT2) mutation. Cardiovasc Res England: 2010. p. 452-60.

25. Hershberger RE, Lindenfeld J, Mestroni L, Seidman CE, Taylor MR, Towbin JA. Genetic evaluation of cardiomyopathy--a Heart Failure Society of America practice guideline. J Card Fail United States: 2009. p. 83-97.

26. Ritter M, Oechslin E, Sutsch G, Attenhofer C, Schneider J, Jenni R. Isolated noncompaction of the myocardium in adults. Mayo Clin Proc 1997; 72: 26-31.

27. Ichida F, Hamamichi Y, Miyawaki T, Ono Y, Kamiya T, Akagi T, et al. Clinical features of isolated noncompaction of the ventricular myocardium: long-term clinical course, hemodynamic properties, and genetic background. J Am Coll Cardiol United States: 1999. p. 233-40.

28. Stollberger C, Finsterer J. A diagnostic dilemma in non-compaction, resulting in near expulsion from the Football World Cup. Eur J Echocardiogr England: 2011.p. E8

29. Jenni R, Oechslin E, Schneider J, Attenhofer Jost C, Kaufmann PA. Echocardiographic and pathoanatomical characteristics of isolated left ventricular non-compaction: a step towards classification as a distinct cardiomyopathy. Heart 2001; 86: 666-71.

30. Jaramillo A, Ramírez A, Galleguillos L, Vallejos J, Illanes S. Derrame isquêmico como a primeira manifestação de hipertrabeculação/não-compactação ventricular grave. Arq Bras Card 2010; 94: e88-e90.

31. Jenni R, Oechslin EN, van der Loo B. Isolated ventricular non-compaction of the myocardium in adults. Heart England: 2007: 11-5.

32.Stollberger C, Finsterer J, Blazek G. Left ventricular hypertrabeculation/ noncompaction and association with additional cardiac abnormalities and neuromuscular disorders. Am J Cardiol United States: 2002: 899-902.

33. Petersen SE, Selvanayagam JB, Wiesmann F, Robson MD, Francis JM, Anderson RH, et al. Left ventricular non-compaction: insights from cardiovascular magnetic resonance imaging. J Am Coll Cardiol United States: 2005: 101-5. 
34. Cheng H, Zhao S, Jiang S, Yu J, Lu M, Ling J, et al. Cardiac magnetic resonance imaging characteristics of isolated left ventricular noncompaction in a Chinese adult Han population. Int J Cardiovasc Imaging 2010.

35. Weiss F, Habermann CR, Lilje C, Razek W, Sievers J, Weil J, et al. [MRI in the diagnosis of non-compacted ventricular myocardium (NCVM) compared to echocardiography]. Rofo 2003; 175: 1214-9.

36. Amir O, Delgado RM, Kar B, Smart FW. The value of cardiac magnetic resonance imaging in the diagnosis of isolated non-compaction of the left ventricle. Isr Med Assoc J 2009; 11: 313-4.

37. Fazio G, Novo G, D'Angelo L, Visconti C, Sutera L, Grassedonio E, et al. Magnetic resonance in isolated noncompaction of the ventricular myocardium. Int J Cardiol 2010; 140: 367-369.

38. Thuny F, Jacquier A, Jop B, Giorgi R, Gaubert JY, Bartoli JM, et al. Assessment of left ventricular non-compaction in adults: side-by-side comparison of cardiac magnetic resonance imaging with echocardiography. Arch Cardiovasc Dis. Netherlands: 2010. p. 150-9.

39. Alhabshan F, Smallhorn JF, Golding F, Musewe N, Freedom RM, Yoo SJ. Extent of myocardial non-compaction: comparison between MRI and echocardiographic evaluation. Pediatr Radiol 2005; 35: 1147-51.

40. McCrohon JA, Richmond DR, Pennell DJ, Mohiaddin RH. Images in cardiovascular medicine. Isolated noncompaction of the myocardium: a rarity or missed diagnosis? Circulation 2002; 106: e22-3.

41. Baumhakel M, Janzen I, Kindermann M, Schneider G, Hennen B, Bohm M. Images in cardiovascular medicine. Cardiac imaging in isolated noncompaction of ventricular myocardium. Circulation 2002; 106: e16-7.

42. Araque Rivadeneira JV, Hidalgo FL. Miocardiopatía no compactada: dos casos clínicos y actualización del tema. Revista argentina de cardiología 2010; 78: $147-150$.

43. Lofiego C, Biagini E, Pasquale F, Ferlito M, Rocchi G, Perugini E, et al. Wide spectrum of presentation and variable outcomes of isolated left ventricular noncompaction. Heart England: 2007. p. 65-71.

44. Kobza R, Jenni R, Erne P, Oechslin E, Duru F. Implantable cardioverterdefibrillators in patients with left ventricular noncompaction. Pacing Clin Electrophysiol United States: 2008. p. 461-7.

45. Epstein AE, DiMarco JP, Ellenbogen KA, Estes NA, Freedman RA, Gettes LS, et al. ACC/AHA/HRS 2008 Guidelines for Device-Based Therapy of Cardiac
Rhythm Abnormalities: a report of the American College of Cardiology/American Heart Association Task Force on Practice Guidelines (Writing Committee to Revise the ACC/AHA/NASPE 2002 Guideline Update for Implantation of Cardiac Pacemakers and Antiarrhythmia Devices) developed in collaboration with the American Association for Thoracic Surgery and Society of Thoracic Surgeons. $J$ Am Coll Cardiol 2008. p. e1-62.

46. Zipes DP, Camm AJ, Borggrefe M, Buxton AE, Chaitman B, Fromer M, et al.ACC/AHA/ESC 2006 guidelines for management of patients with ventricular arrhythmias and the prevention of sudden cardiac death: a report of the American College of Cardiology/American Heart Association Task Force and the European Society of Cardiology Committee for Practice Guidelines (Writing Committee to Develop Guidelines for Management of Patients With Ventricular Arrhythmias and the Prevention of Sudden Cardiac Death). J Am Coll Cardiol 2006. p. e247-346.

47. Taniguchi M, Hioka T, Maekawa K, Takagagi K, Shoji K, Yoshida K. Adult case of isolated ventricular noncompaction discovered by complete atrioventricular block. Circ J Japan 2004. p. 873-5.

48. Sajeev CG, Francis J, Shanker V, Vasudev B, Abdul Khader S, Venugopal K. Young male with isolated noncompaction of the ventricular myocardium presenting with atrial fibrillation and complete heart block. Int J Cardiol 2006. p. 142-3.

49. Yildiz A, Ozeke O, Akyol S, Demirbag R, Yilmaz R, Gur M. Biventricular myocardial noncompaction presenting with complete atrioventricular block. Int J Cardiol 2009. p. e34-6.

50. Banci M, Martinoli R, Dofcaci A, Piccirilli S, Papetti F, Sansoni I, et al. A Case of Isolated Left Ventricular Noncompaction with Basal ECG-Tracing Strongly Suggestive for Type-2 Brugada Syndrome. Cardiol Res Pract 2010; 2011: 201962.

51. Ogawa K, Nakamura Y, Terano K, Ando T, Hishitani T, Hoshino K. Isolated non-compaction of the ventricular myocardium associated with long QT syndrome: a report of 2 cases. Circ J Japan 2009. p. 2169-72.

52. Villacorta H, Sampaio JM, Santos FBAD, Carrera V, Pereira CC, Mesquita ET. Insuficiência cardiaca refratária em um paciente portador de miocárdio não compactado. Arquivos Brasileiros de Cardiologia. 2009; 93: e93-e96.

53. Correia E, Santos LF, Rodrigues B, Gama P, Cabral C, Santos O. Miocárdio não compactado em paciente com infarto agudo do miocárdio. Arquivos Brasileiros de Cardiologia. 2010; 94: e125-e127.

54. Restrepo G, Castaño RI, Mármol A. Ventrículo izquierdo no compacto/ventrículo izquierdo hipertrabeculado. Revista Colombiana de Cardiología 2005; 12: 20-24 